Conference Proceedings Paper

\title{
Personalized Nutrition Plans based on Measurement of Specific Redox Biomarkers in Human Blood Firstname
}

\section{Dimitrios Koureta}

1 Department of Biochemistry-Biotechnology , University of Thessaly, Larissa, Greece

* Correspondence: dkouret@uth.gr

Published: 3 December 2020

\begin{abstract}
One of the most studied question in modern history is the particular way of bridging the gap between lifespan and healthspan? Even as average life expectancy has increased, there remains a sizeable gap between life span and health span - the years an individual lives without disease. The second question we must answer is the possibility that our lifespan and/or healthspan is "Programmed" in our genes? Or maybe there are other parameters that contribute to this model? Genome Analysis with "single nucleotide polymorphisms" (SNPs) of exceptionally long-lived people reveal $10-15 \%$ complex genetic signatures and very few genes consistently involved. The rest refers to the 3 pillars of metabolic health, the diet, the exercise, and the use of fasting in our everyday plan. Nutrient influence on healthy aging is being extensively studied in humans and in many animal models of aging. Moreover, the latest evidence is showing that regular physical activity can actually slow the aging process on a cellular level and potentially add years to your life. Additionally, intermittent fasting elicits evolutionarily conserved, adaptive cellular responses that are integrated between and within organs in a manner that improves glucose regulation, increases stress resistance, and suppresses inflammation. All these measurements proposed by our research team provides a holistic approach for the evaluation of redox status parameters for several conditions. Therefore, the effect of personalized nutrition on human redox status is evaluated and human health is improved.
\end{abstract}

